\title{
Simulation of Microbial Depolymerization Processes of Polyethylene Glycol based on Degradation Rate after Cultivation for Two and Four Days
}

\author{
Masaji Watanabe \\ Graduate School of Environmental and Life Science \\ Okayama University \\ Okayama, Japan \\ E-mail: watanabe@ems.okayama-u.ac.jp
}

\author{
Fusako Kawai \\ Center for Nanomaterials and Devices \\ Kyoto Institute of Technology \\ Kyoto, Japan \\ fkawai@kit.ac.jp
}

\begin{abstract}
Microbial depolymerization processes of polyethylene glycol are analyzed by numerical simulation. The weight distribution after cultivation of microorganisms for two and four days was used to solve an inverse problem for the molecular factor of the degradation rate. Numerical results are compared with experimental results to determine the time factor of the degradation rate. The transition of the weight distribution was simulated by solving an initial value problem.
\end{abstract}

Keywords- Microbial depolymerization; Mathematical modeling; Numerical simulation; Polyethylene glycol

\section{INTRODUCTION}

Microbial depolymerization processes are divided into exogenous type processes and endogenous type processes. Molecules are depolymerized by liberating monomers from their terminals in an exogenous type depolymerization process. Polyethylene (PE) is a polymer depolymerized in exogenous type depolymerization processes. A mathematical model was proposed, and implemented for simulations of PE biodegradation processes [1].

Polyethylene glycol (PEG) is also a polymer depolymerized in exogenous depolymerization processes. PEG is one of polyethers expressed chemically by $\mathrm{HO}(\mathrm{R}-$ O) ${ }_{\mathrm{n}} \mathrm{H}$, e.g., PEG: $\mathrm{R}=\mathrm{CH}_{2} \mathrm{CH}_{2}$, PPG: $\mathrm{R}=\mathrm{CH}_{3} \mathrm{CHCH}_{2}$, PTMG: $\mathrm{R}=\left(\mathrm{CH}_{2}\right)_{4}$ [2]. PEG molecules are depolymerized by liberation of $\mathrm{C}_{2}$ compounds from their terminals [3, 4]. Microbial consortium E1 was cultivated in a culture medium, in which the only carbon source was PEG, and the weight distribution before and after the cultivation was compared. Figures 1 shows the transition of the weight distribution of PEG6000 for seven days.

Techniques developed for the PE biodegradation were extended to studies of the exogenous depolymerization processes of PEG. Inverse problems were formulated for molecular factors of degradation rates based on the weight distribution of PEG with respect to the molecular weight before and after cultivation of a microbial consortium E1. Initial value problems were solved to simulate the transition of the weight distribution. The time factors of degradation rates were also considered in studies on depolymerization processes of PEG [5]. A model originally proposed for endogenous depolymerization processes was reformulated to analyze the exogenous depolymerization processes of PEG [6] and PE [7]. Techniques developed for the analysis of PE biodegradation [7] was extended to studies on exogenous depolymerization processes of PEG [8].

In this study, numerical results were obtained for a microbial depolymerization process of PEG6000. A molecular factor of a degradation rate was obtained from the weight distribution of PEG6000 after cultivation of the microbial consortium E1 for two and four days. The initial value problem was solved to simulate the transition of the weight distribution of PEG6000.

\section{MODEL FOR MICROBIAL DEPOLYMERIZATION PROCESSES OF EXOGENOUS TYPE}

Molecules of a single polymer all have the same chemical structure, but their molecular weight differ from one molecule to another. Let $w(t, M)$ be the weight distribution of molecules with respect to the molecular weight $M$ at time $t$. A molecule of molecular weight $M$ becomes a molecule of molecular weight $K$ when a part of molecular weight $M-K$ is liberated. That leads to a weight shift from $w(t, M)$ to $w(t, K)$. Let $q(K, M)$ be the decrease in $w(t, M)$ per unit weight in the weight shift from $w(t, M)$ to $w(t, M)$. In other words, $q(K, M)$ is the probability of the event in which a molecule with molecular weight $K$ is produced from a molecule with molecular weight $M$. The weight distribution $w(t, M)$ satisfies the equation $(1)[7,8]$.

$$
\begin{aligned}
\frac{\partial w}{\partial t}= & -\gamma(t, M) w \\
& +\int_{M}^{\infty} \frac{M}{K} \gamma(t, K) q(M, K) w(t, K) d K .
\end{aligned}
$$

Given an initial weight distribution $f(M)$, equation (1) and the initial condition

$$
w(0, M)=f(M)
$$

form an initial value problem to solve for $w(t, M)$ provided the degradation rate $\gamma(t, M)$ is known.

Time factors of degradability such as temperature, dissolved oxygen, and microbial population act evenly on molecules regardless of molecular sizes. For such factors, the degradation rate $\gamma(t, M)$ is a product of a function of $t$, which we denote by $\sigma(t)$, and a function of $M$ which we denote by $\lambda(M)$. Substitution $\gamma(t, M)=\sigma(t) \lambda(M)$ in the equation (1) leads to 


$$
\begin{aligned}
\frac{\partial w}{\partial t}=- & \sigma(t)\{\lambda(M) w \\
& \left.+\int_{M}^{\infty} \frac{M}{K} \lambda(K) q(M, K) w(t, K) d K\right\} .
\end{aligned}
$$

The memoryless behavior in an exogenous depolymerization processes leads to the exponential distribution $\rho e^{-\rho J}$ for the amount $J$ liberated from a molecule [9]. In an exogenous depolymerization process, amount $M-K$ is separated from a molecule with molecular weight $M$ to become a molecule with molecular weight $K$, so that $q(K, M)=\rho e^{-\rho(M-K)}$. Equation (3) now becomes

$$
\begin{aligned}
\frac{\partial w}{\partial t}=- & \sigma(t)\{\lambda(M) w \\
& \left.+c(M) \int_{M}^{\infty} \lambda(K) d(K) w(t, K) d K\right\},
\end{aligned}
$$

where

$$
c(M)=\rho M e^{\rho M}, \quad d(K)=\frac{1}{K} e^{-\rho K} .
$$

In an exogenous type depolymerization process, molecules depolymerized by successive separations of monomers. Let $L$ be the molecular weight of a monomer liberated from a molecule in one cycle of a depolymerization process, e.g. PE: $L=28\left(\mathrm{CH}_{2} \mathrm{CH}_{2}\right)$, PEG: $L=44\left(\mathrm{CH}_{2} \mathrm{CH}_{2} \mathrm{O}\right)$. The intensity $\rho$ is given by the expression $(6)[7,8]$.

$$
\rho=\frac{\log 2}{L} \text {. }
$$

Mathematical model (7) for the time evolution of the microbial population was proposed in previous studies [5];

$$
\frac{d \sigma}{d t}=k\left(1-h \frac{1}{\int_{0}^{\infty} \lambda(M) w(t, M) d M}\right) \sigma
$$

where $k$ and $h$ are positive constants. The equation (7) is associated with the initial condition

$$
\sigma(0)=\sigma_{0} \text {. }
$$

where $\sigma_{0}$ is the initial microbial population. Given the time factor and the molecular factor of the degradation rate, the initial value problem (4), (7), (2), (8) were solved to simulate the microbial depolymerization processes $[10,11,12,13,14]$.

\section{REDUCTION TO A MODEL WITH TIME INDEPENDENT DEGRADATION RATE AND INVERSE PROBLEM FOR THE MOLECULAR FACTTOR}

Define the change of variables from $t$ to $\tau$,

$$
\tau=\int_{0}^{t} \sigma(s) d s,
$$

and let $W(\tau, M)=w(t, M)$. Equation (4) leads to

$$
\frac{\partial W}{\partial \tau}=-\lambda(M) W+c(M) \int_{M}^{\infty} \lambda(K) d(K) W(\tau, K) d K .
$$

Given the molecular factor $\lambda(M)$ of the degradation rate, equation (10) and the initial condition

$$
W\left(\mathrm{~T}_{1}, M\right)=f(M)
$$

form an initial value problem. Equation (10), the initial condition (11) and the condition

$$
W\left(\mathrm{~T}_{2}, M\right)=g(M)
$$

form an inverse problem for the molecular factor $\lambda(M)$ for which the solution of the initial value problem (10) and (11) also satisfies the condition (12).

Techniques developed in previous studies $[7,8]$ were applied to solve the inverse problem for the molecular factor $\lambda(M)$ of the degradation rate. The weight distribution functions after the cultivation of the microbial consortium E1 for two days and four days were set as the initial condition and the final condition, respectively, and the inverse problem (10), (11), (12) was solved numerically with $c(M)$ and $d(K)$ given by the expressions (5) to obtain the molecular factor of the degradation rate. Figure 2 shows a numerical result. Figure 3 shows the profiles of $W(\tau, M)$ obtained by solving the initial value problem (10), (13), where $f(M)$ is the initial weight distribution shown in the figure 1.

$$
W(0, M)=f(M)
$$

\section{SimUlation WITH TIME FACTOR}

Recall that $\sigma(t)$ is a part of the solution of the initial value problem (4), (7), (2), (8). Let $S(\tau)=\sigma(t)$, where the equation (9) holds for $t$ and $\tau$. In view of the equation (9), $t=q(\tau)$, where

$$
q(\tau)=\int_{0}^{\tau} \frac{1}{S(r)} d r,
$$

and $S(\tau)$ is a solution of the initial value problem

$$
\frac{d S}{d \tau}=k\left(1-h \frac{1}{\int_{0}^{\infty} \lambda(M) W(\tau, M) d M}\right), \quad S(0)=\sigma_{0}
$$

Note that $q(\tau)$ is also a function of $\sigma_{0}, k$, and $h$. Results shown in Figures 3 lead to the system of three equations for $\sigma_{0}, k$, and $h$.

$$
q(1)=2, \quad q(3)=4, \quad q(22)=7 .
$$

A numerical technique developed in a previous study [13] is applied to the system of equations (16) to solve for $\sigma_{0}, k$, and $h$ numerically, and the following values were obtained.

$$
\begin{aligned}
& \sigma_{0}=0.357101644350559, \quad k=0.486446928261632, \\
& h=7.72797586485759 .
\end{aligned}
$$

Using those parameter values, the initial value problem (4), (7), (2), (8) was solved numerically. Figures 4 shows numerical results and the experimental results.

\section{CONCLUSION}

The inverse problem for the molecular factor of the degradation rate was solved for the weight distribution before and after cultivation of the microbial consortium E1 on PEG for three days, and the initial value problem (4), (7), 
(2), (8) was solved numerically for various values of the parameters which characterize the time factor of the degradation rate $[10,11,12,13]$. The inverse problem for the molecular factor of the degradation rate was solved for the weight distribution before and after cultivation of the microbial consortium E1 on PEG6000 for two days [14].

In this study, the inverse problem was solved for the weight distribution after cultivation of microbial consortium E1 on PEG6000I after two days and four days. Our numerical results show that our techniques are applicable to a wide range of experimental data.

\section{ACKNOWLEDGMENT}

The authors thank Ms. Y. Shimizu for her technical support. This work was supported by JSPS KAKENHI Grant Number 24540127.

\section{REFERENCES}

[1] Masaji Watanabe, Fusako Kawai, Masaru Shibata, Shigeo Yokoyama, Yasuhiro Sudate, Shizue Hayashi, Analytical and computational techniques for exogenous depolymerization of xenobiotic polymers, $\begin{array}{lllll}\text { Mathematical } & \text { Biosciences } & 192 & \text { (2004) } & \text { 19-37. }\end{array}$ doi:10.1016/j.mbs.2004.06.006

[2] F. Kawai, Biodegradability and chemical structure of polyethers, Kobunshi Ronbunshu, 50, 10, 775--780, 1993, In Japanese.

[3] F. Kawai, Breakdown of plastics and polymers by microorganisms, Advances in Biochemical Engineering/Biotechnology, 52, 151--194, 1995.

[4 F. Kawai, Microbial degradation of polyethers, Applied Microbiology and Biotechnology, 58, 30--38, 2002. doi: 10.1007/s00253-001-08502

[5] M. Watanabe and F. Kawai, Effects of microbial population in degradation process of xenobiotic polymers, In P. Howlett, M. Nelson, and A. J. Roberts, editors, Proceedings of the 9th Biennial Engineering Mathematics and Applications Conference, EMAC-2009, volume 51 of ANZIAM J., pages C682--C696, September 2010. http://journal.austms.org.au/ojs/index.php/ANZIAMJ/article/view/24 33

[6] M. Watanabe and F. Kawai, Study of biodegradation of xenobiotic polymers with change of microbial population, In W. McLean and A. J. Roberts, editors, Proceedings of the 15th Biennial Computational Techniques and Applications Conference, CTAC-2010, volume 52 of ANZIAM J., pages C410--C429, July 2011.

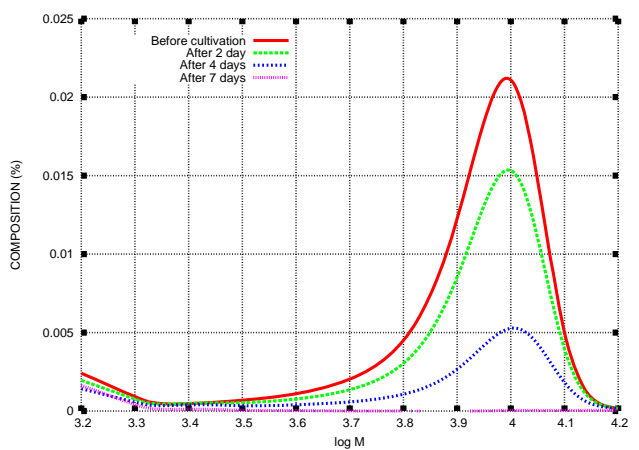

http://journal.austms.org.au/ojs/index.php/ANZIAMJ/article/view/39 65.

[7] Watanabe M, Kawai F (2012) Modeling Biodegradation of Polyethylene with Memoryless Behavior of Metabolic Consumption, J Bioremed Biodegrad 3: 146. doi:10.4172/2155-6199.1000146.

[8] M. Watanabe and F. Kawai, Study on microbial depolymerization processes of xenobiotic polymers with mathematical modelling and numerical simulation, In Mark Nelson, Mary Coupland, Harvinder Sidhu, Tara Hamilton, and A. J. Roberts, editors, Proceedings of the 10th Biennial Engineering Mathematics and Applications Conference, EMAC-2011, volume 53 of ANZIAM J., pages C203--C217, June 2012

http://journal.austms.org.au/ojs/index.php/ANZIAMJ/article/view/51 07

[9] Manfred Denker and Wojbor A. Woyczyński, Introductory Statistics and Random Phenomena, Uncertainty, Complexity and Chaotic Behavior in Engineering and Science, with Mathematica $\mathbb{R}$ Uncertain Virtual Worlds ${ }^{\text {TM }}$ by Bernard Ycart, Birkh user, Boston, 1998.

[10] MASAJI WATANABE, FUSAKO KAWAI, Computational Study of Microbial Depolymerization Processes of Xenobiotic Polymers, ADVANCES IN COMPUTER SCIENCE, Prodeedings of the 6th WSEAS European Computing Conference (ECC'12), Prague, Czech Republic, September 24-26, 2012, Editor Sumanth Yenduri, Recent Advances in Computer Engineering Series 5, Published by WSEAS Press, 128-133.

[11] Masaji Watanabe, Fusako Kawai, Computational Study on Effects of Microbial Population in Biodegradation of Xenobiotic Polymers, PROCEEDINGS, 2012 International Conference of Information Science and Computer Applications (ICISCA 2012), November 19th20th, 2012, Bali Dynasty Resort, Bali-Indonesia, Advances in Information Technology and Applied Computing, Volume (1), 273 278.

[12] MASAJI WATANABE, FUSAKO KAWAI, Numerical Study on Microbial Effects in Biodegradation of Xenobiotic Polymers, Editors, Hamido Fujita, Milan Tuba, Jun Sasaki, Recent Advances in Automatic Control, Modelling \& Simulation, Proceedings of the 12th International Conference on System Science and Simulation in Engineering (ICOSSSE'13), Morioka City, Iwate, Japan, April 23-25, 2013, Recent Advances in Electrical Engineering Series | 12, 121 126, ISSN: 1790-5117, ISBN: 978-1-61804-177-7

[13] Masaji Watanabe, Fusako Kawai, STUDY ON MICROBIAL DEPOLYMERIZATION PROCESSES OF EXOGENOUS TYPE BY MODELING AND SIMULATION, Proceedings of the IASTED International Conference on Control and Applications, Editor: M.H. Hamza, August 26 - 28, 2013 Honolulu, USA, ISBN: 978-0-88986958-5, DOI:10.2316/P.2013.805-033

[14] Masaji Watanabe, Fusako Kawai, Simulaltion for microbial depolymerization processes of polyethylene glycol, Submitted.

Figure 1. Weight distribution of PEG 6000 before and after cultivation of the microbial consortium E1 for seven days [18]. 


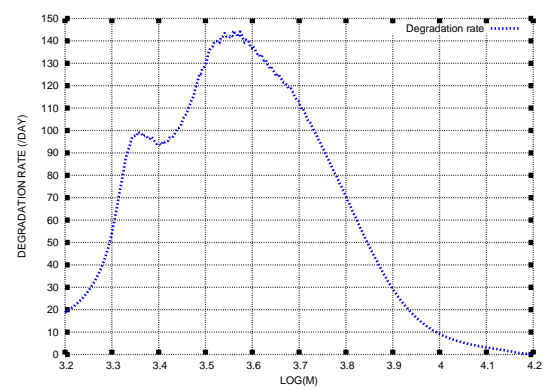

Figure 2. Degaradation rate based on the weight distribution after cultivation of the microbial consortium E1 for two and four days.
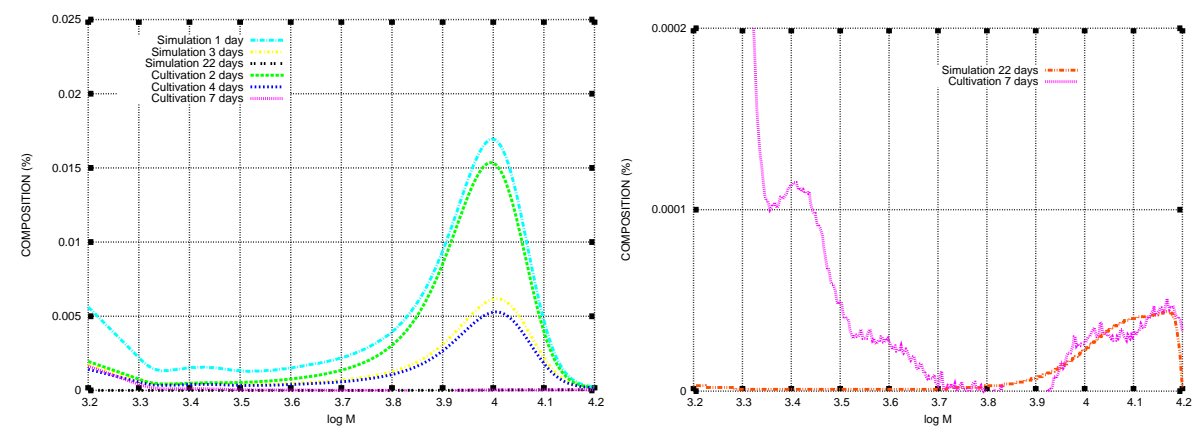

Figure 3. Numerical results for the initial value problem (10), (13) and experimenatal results for the weight distribution of PEG after cultivation of microbial consortium E1 for two, four, and seven days.
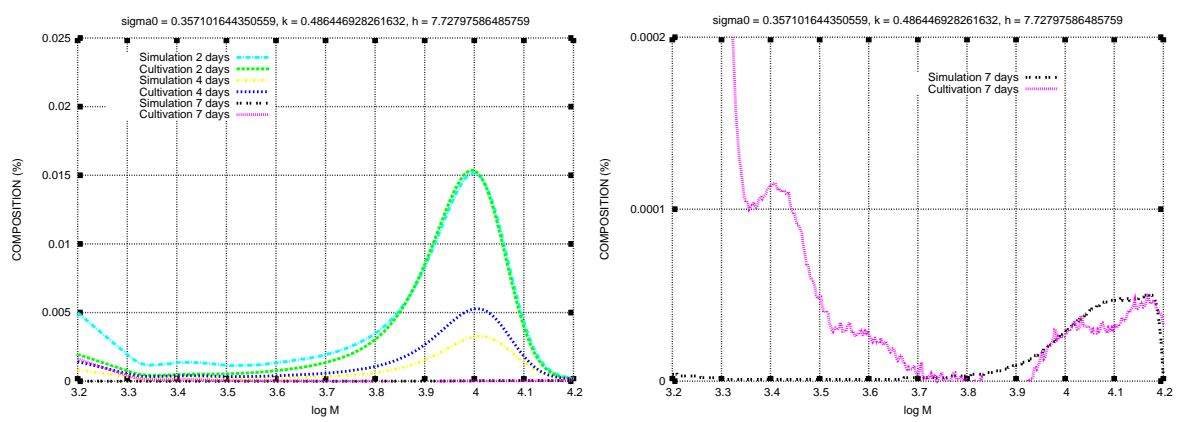

Figure 4. Numerical results for the initial value problem (4), (7), (2), (8) and experimenatal results for the weight distribution of PEG after cultivation of microbial consortium E1 for two, four, and seven days. 